\title{
Precessing Ball Solitons in Kinetics of a Spin-Flop Phase Transition
}

\author{
V. V. Nietz \\ Joint Institute for Nuclear Research, Dubna, Moscow 141980, Russia \\ Correspondence should be addressed to V. V. Nietz, nietz@jinr.ru \\ Received 25 April 2011; Accepted 17 May 2011 \\ Academic Editor: Frank Tsui
}

Copyright () 2011 V. V. Nietz. This is an open access article distributed under the Creative Commons Attribution License, which permits unrestricted use, distribution, and reproduction in any medium, provided the original work is properly cited.

The fundamentals of precessing ball solitons (PBS), arising as a result of the energy fluctuations during spin-flop phase transition induced by a magnetic field in antiferromagnets with uniaxial anisotropy, are presented. The PBS conditions exist within a wide range of amplitudes and energies, including negative energies relative to an initial condition. For each value of the magnetic field, there exists a precession frequency for which a curve of PBS energy passes through a zero value (in bifurcation point), and hence, in the vicinity of this point the PBS originate with the highest probability. The characteristics of PBS, including the time dependences of configuration, energy, and precession frequency, are considered. As a result of dissipation, the PBS transform into the macroscopic domains of a new phase.

\section{Introduction}

Magnetic solitons with spherical symmetry, which can arise in crystals with magnetic ordering during the phase transitions induced by a magnetic field, were considered in some papers [1-5]. The cases when it is possible to confine oneself to uniaxial symmetry of the crystal are of particular interest. For such a crystal, in addition to the amplitude (with corresponding configuration) and pulse, the solitons have the third parameter: the frequency of precession. In these cases, each value of an external field relates to a continuous spectrum of solitons by frequency and corresponding energy. Near boundaries of metastability, for each value of a field, the spectrum of precessing ball solitons (PBS) has the bifurcation point where the probability of PBS spontaneous origin is increasing abnormally. The frequency dependencies of energy and PBS configuration, as well as the process of PBS spontaneous origin at spin-flop transition in antiferromagnets have been discussed in [5]. However, the analysis of time evolution connected with dissipation of energy in PBS is not correct in this article.

In the present article, it is shown that dissipation of energy is accompanied not only by the change of configuration of solitons but also by the change of the precession frequency. Taking into account this time dependency, we can carry out a more comprehensive analysis of the quantities of PBS and to consider the whole process of transformation of PBS into macroscopic domains of a new phase.

In the first chapter, we represent a more correct analysis of equations and expressions for PBS than in $[4,5]$. In the second one, the character of the time change of PBS that connected with dissipation is shown. In the third chapter, the influence of the movement on the form of PBS is discussed. Finally, in the fourth chapter, we estimate the influence of the demagnetizing fields.

\section{Equations for Precessing Ball Solitons (PBS)}

To analyze magnetic solitons in an antiferromagnet with uniaxial anisotropy, we used the following expression for the thermodynamic potential:

$$
\begin{aligned}
W= & -\frac{A}{2} \mathbf{l}^{2}+\frac{B}{2} \mathbf{m}^{2}+\frac{C}{4}(\mathbf{m l})^{2}+\frac{K_{1}}{2}\left(\left|m_{\perp}\right|^{2}+\left|l_{\perp}\right|^{2}\right) \\
& -\frac{K_{2}}{4}\left(\left|m_{\perp}\right|^{2}+\left|l_{\perp}\right|^{2}\right)^{2}-m_{z} H+\frac{\alpha_{x y}}{2}
\end{aligned}
$$




$$
\begin{aligned}
& \times\left[\left(\frac{\partial \mathbf{m}}{\partial X}\right)^{2}+\left(\frac{\partial \mathbf{m}}{\partial Y}\right)^{2}+\left(\frac{\partial \mathbf{l}}{\partial X}\right)^{2}+\left(\frac{\partial \mathbf{l}}{\partial Y}\right)^{2}\right] \\
& +\frac{\alpha_{z}}{2}\left[\left(\frac{\partial \mathbf{m}}{\partial Z}\right)^{2}+\left(\frac{\partial \mathbf{l}}{\partial Z}\right)^{2}\right] .
\end{aligned}
$$

Here $\mathbf{m}$ and $\mathbf{1}$ are nondimensional ferromagnetism and antiferromagnetism vectors; $l_{\perp}=l_{x}+i l_{y}, \quad m_{\perp}=m_{x}+i m_{y}$; the absolute value of the vector $\mathbf{l}$ at $H=0$ equals $1 ; K_{1}>$ $0, \quad K_{2}>0$; magnetic field $H$ is directed along the anisotropy axis $Z$.

The equations of motion for the $\mathbf{l}$ and $\mathbf{m}$ vectors, taking into account the energy dissipation, are

$$
\begin{aligned}
\frac{\partial \mathbf{l}}{\partial t} & =\frac{2 \mu_{B}}{\hbar}\left(\mathbf{m} \times \frac{\delta W}{\delta \mathbf{l}}+\mathbf{l} \times \frac{\delta W}{\delta \mathbf{m}}\right)+\Gamma\left(\mathbf{m} \times \frac{\partial \mathbf{l}}{\partial t}+\mathbf{l} \times \frac{\partial \mathbf{m}}{\partial t}\right), \\
\frac{\partial \mathbf{m}}{\partial t} & =\frac{2 \mu_{B}}{\hbar}\left(\mathbf{m} \times \frac{\delta W}{\delta \mathbf{m}}+\mathbf{l} \times \frac{\delta W}{\delta \mathbf{l}}\right)+\Gamma\left(\mathbf{m} \times \frac{\partial \mathbf{m}}{\partial t}+\mathbf{l} \times \frac{\partial \mathbf{l}}{\partial t}\right) .
\end{aligned}
$$
form:

The solutions of (2) can be presented in the following

$$
\begin{gathered}
l_{\perp}(\mathbf{r}, \tau)=q(\mathbf{r}, \tau) e^{i(\omega(\tau) \tau-k(\tau) x)}, \\
m_{\perp}(\mathbf{r}, \tau)=p(\mathbf{r}, \tau) e^{i(\omega(\tau) \tau-k(\tau) x)} .
\end{gathered}
$$

For the simplicity, it is supposed that the excitations advance along the $x$-axis. The time dependencies of the $\omega$ and $k$ values are necessary for analyzing the time evolution of PBS.

From (2), we have the following system of equations:

$$
\begin{aligned}
k_{1}^{-0.5}(\omega+\Delta+h) q= & -\left(1-\frac{k_{2}}{k_{1}}\left(q^{2}+p^{2}\right)+k^{2}\right)\left(m_{z} q+l_{z} p\right) \\
& +\frac{a}{k_{1}}\left(m_{z} q-l_{z} p\right)+\frac{1}{k_{1}}\left(m_{z} q-l_{z} p\right) \\
& +m_{z} \Delta q-q \Delta m_{z}+l_{z} \Delta p-p \Delta l_{z}-Q A_{1}
\end{aligned}
$$

$$
\begin{aligned}
k_{1}^{-0.5}(\omega+\Delta+h) p= & -\left(1-\frac{k_{2}}{k_{1}}\left(q^{2}+p^{2}\right)+k^{2}\right)\left(l_{z} q+m_{z} p\right) \\
& +l_{z} \Delta q-q \Delta l_{z}+m_{z} \Delta p-p \Delta m_{z}-Q A_{2}
\end{aligned}
$$

$$
\begin{aligned}
& \frac{\partial p}{\partial \tau}=\sqrt{k_{1}} {\left[2 k\left(l_{z} \frac{\partial q}{\partial x}+m_{z} \frac{\partial p}{\partial x}\right)\right.} \\
&\left.-Q(\omega+\Delta)\left(l_{z} q+m_{z} p\right)\right], \\
& \frac{\partial q}{\partial \tau}=\sqrt{k_{1}} {\left[2 k\left(m_{z} \frac{\partial q}{\partial x}+l_{z} \frac{\partial p}{\partial x}\right)\right.} \\
&\left.-Q(\omega+\Delta)\left(m_{z} q+l_{z} p\right)\right],
\end{aligned}
$$

$$
\frac{\partial l_{z}}{\partial \tau}=2 \sqrt{k_{1}}\left[k\left(p \frac{\partial q}{\partial x}+q \frac{\partial p}{\partial x}\right)+Q(\omega+\Delta) q p\right]
$$

$$
\frac{\partial m_{z}}{\partial \tau}=-\sqrt{k_{1}}\left[k \frac{d}{d x}\left(q^{2}+p^{2}\right)\right.
$$

$$
\left.-Q(\omega+\Delta)\left(q^{2}+p^{2}\right)\right]
$$

where $\Delta=((d \omega / d \tau) \tau-(d k / d \tau) x)$ and

$$
\begin{aligned}
& A_{1}=\left(q \frac{\partial m_{z}}{\partial \tau}-m_{z} \frac{\partial q}{\partial \tau}+p \frac{\partial l_{z}}{\partial \tau}-l_{z} \frac{\partial p}{\partial \tau}\right), \\
& A_{2}=\left(q \frac{\partial l_{z}}{\partial \tau}-l_{z} \frac{\partial q}{\partial \tau}+p \frac{\partial m_{z}}{\partial \tau}-m_{z} \frac{\partial p}{\partial \tau}\right) .
\end{aligned}
$$

In (3)-(11), $h=H B^{-0.5} K_{1}^{-0.5}, k_{1}=K_{1} / B, k_{2}=K_{2} / B, a=$ $A / B, \quad c=C / B$ and the dimensionless coordinates and time are used, $x=K_{1}^{0.5} \alpha_{x y}^{-0.5} X, y=K_{1}^{0.5} \alpha_{x y}^{-0.5} Y, z=K_{1}^{0.5} \alpha_{z}^{-0.5} Z$; $\tau=2 \mu_{B}\left(K_{1} B\right)^{0.5} \hbar^{-1} t ; Q=\Gamma / \sqrt{k_{1}}$.

First of all, take notice that inserting (6)-(9) into (4) and (5), we can obtain a system of two modified equations describing the soliton configuration but not containing the evident time dependency. However, the time dependency of the parameters $p, q, l_{z}$, and $m_{z}$ characterizing the soliton form and described by (6)-(9), is remaining. It means that during the soliton evolution connected with energy dissipation not only the configuration of PBS but also the frequency of precession $\omega$ and $k$ value are changing too. For each time moment, the definite configuration of PBS, frequency $\omega$, and wave value $k$ correspond. The correspondence between the changing values of $\omega, k$, and configuration is explicitly defined by such modified equations (4)-(5).

To analyze the approximate soliton solutions of (4)(9) system, it is convenient to transform this system into one equation. First of all, we will receive expression for magnetization that depends on $q$ and $p$. The relaxation time of ferromagnetic moment is less for some orders than the relaxation time for the $\mathbf{l}$ vector. Therefore, for $m_{z}$ component we use its quasiequilibrium value, which can be obtained from $\delta W / \delta m_{z}=0$ (see [5])

$$
\begin{aligned}
m_{z} \cong m_{z 0} & =\frac{1}{B+C l_{z}^{2}}\left[H_{z}-\frac{C}{2} l_{z}\left(l_{\perp} m_{\perp}^{*}+l_{\perp}^{*} m_{\perp}\right)\right] \\
& =\frac{\delta \sqrt{k_{1}} h-(1-\delta) l_{z} p q}{l_{z}^{2}+\delta\left(1-l_{z}^{2}\right)} \\
& \cong-\frac{p q}{l_{z}}+\frac{\delta}{l_{z}^{2}}\left(\sqrt{k_{1}} h+\frac{p q}{l_{z}}\right),
\end{aligned}
$$

where $\delta=B /(C+B)=\chi_{\mathrm{II}} / \chi_{\perp}$ is the ratio of two magnetic susceptibilities.

Taking into consideration that $k_{1} \ll 1, k_{2}\left(q^{2}+p^{2}\right) \ll 1$ in (5) and for long-wave oscillations $k_{1} \Delta q \ll q, k_{1} \Delta l_{z} \ll l_{z}$, 
$k_{1} \Delta m_{z} \ll m_{z}, k_{1} \Delta p \ll p$, from (4) and (13) we obtain the following dependence

$$
\begin{aligned}
p \cong & -\frac{\sqrt{\mathrm{k}_{1}}(\omega+\Delta+h) q l_{z}}{q^{2}+l_{z}^{2}}\left[1-a\left(2 q^{2}-1\right)\right] \\
& +\delta(1+a) \sqrt{k_{1}} \frac{q}{l_{z}}\left(h-(\omega+\Delta+h) q^{2}\right) \\
& -k_{1} \sqrt{k_{1}} k^{2}(\omega+\Delta+h) q l_{z}\left(2 q^{2}-1\right)-Q l_{z} A_{1}
\end{aligned}
$$

Using the (13) correlation, from (5) we obtain the following equation for PBS:

$$
\begin{aligned}
\Delta q- & \frac{q}{l_{z}} \Delta l_{z} \\
= & {\left[1-(1+a)(\omega+\Delta+h)^{2}+k^{2}+\delta \frac{(1+a)(\omega+\Delta+h) h}{l_{z}^{2}}\right] } \\
& \times q-\left[\frac{k_{2}}{k_{1}}-2 a \times(\omega+\Delta+h)^{2}+2 k_{1}(\omega+\Delta+h)^{2} k^{2}\right. \\
& \left.+\delta(1+a) \frac{(\omega+\Delta+h)^{2}}{l_{z}^{2}}\right] q^{3}+Q \frac{A_{2}}{l_{z}} .
\end{aligned}
$$

Let's confine ourselves to low temperature approximation, that is, suppose that $(\operatorname{lm})=0$, in such case $a=\delta=0$, $q^{2}+p^{2}+l_{z}^{2}+m_{z}^{2}=1$,

$$
\begin{aligned}
A_{1}=2 \sqrt{k_{1}} k\left[3 q p \frac{\partial q}{\partial x}+\left(2 q^{2}+p^{2}-1\right) \frac{\partial p}{\partial x}\right. \\
\left.-\frac{1}{2} p \frac{d}{d x}\left(q^{2}+p^{2}\right)\right]+Q(\omega+\Delta) p, \\
A_{2}=2 \sqrt{k_{1}} k\left[3 p q \frac{\partial p}{\partial x}+\left(2 p^{2}+q^{2}-1\right) \frac{\partial q}{\partial x}\right. \\
\left.-\frac{1}{2} q \frac{d}{d x}\left(q^{2}+p^{2}\right)\right]+Q(\omega+\Delta) q,
\end{aligned}
$$

and the equation for PBS is as follows:

$$
\begin{aligned}
\Delta q-\frac{q}{l_{z}} \Delta l_{z}= & \left(1-(\omega+\Delta+h)^{2}+k^{2}\right) q \\
& -\left(\frac{k_{2}}{k_{1}}+2 k_{1}(\omega+\Delta+h)^{2} k^{2}\right) q^{3}+Q \frac{A_{2}}{l_{z}}
\end{aligned}
$$

This equation should be supplemented by the following expression obtained from (7), (12), (13) at $a=\delta=0$ and describing the evolution of PBS:

$$
\begin{aligned}
\frac{\partial q}{\partial \tau} \cong & k_{1}(\omega+\Delta+h) \\
& \times\left[2 k\left(3 q^{2}-1\right) \frac{\partial q}{\partial x}-Q(\omega+\Delta) q\left(2 q^{2}-1\right)\right] .
\end{aligned}
$$

Only in the case of immovable PBS, that is, at $k \equiv 0,(17)$ has the solutions with a spherical symmetry. For such case, the equation has the following form:

$$
\begin{aligned}
& \frac{d^{2} q}{d r^{2}}+\frac{2}{r} \frac{d q}{d r}+\frac{q}{1-q^{2}}\left(\frac{d q}{d r}\right)^{2} \\
& =q\left[\left(1-q^{2}\right)\left(1-(\omega+h)^{2}-\frac{k_{2}}{k_{1}} q^{2}\right)+Q^{2} \omega\left(1+\frac{1}{2} q^{2}\right)\right]
\end{aligned}
$$

(taking into account that $l_{z}^{2} \cong 1-q^{2}$ ). In this equation, the frequency $\omega$, as well as $q$ values, depends on time. Thus we make the replacement $(\omega+(d \omega / d \tau) \tau) \rightarrow \omega(\tau)$, which is possible considering a rather slow change of the precession frequency in comparison with the change of precession phase. Note that in (19), the addition connected with the dissipation is negligibly small, $Q^{2}|\omega| \ll 1$ (in our examples $Q=0.02,|\omega|<0.03)$. Therefore, in further calculations of PBS configuration we will neglect this addition.

Transforming (19) with respect to $l_{z}$ parameter, we can obtain the following equation that can be used to analyze the PBS originated during the reverse phase transition, at decrease of the field:

$$
\begin{aligned}
& \frac{d^{2} l_{z}}{d r^{2}}+\frac{2}{r} \frac{d l_{z}}{d r}+\frac{l_{z}}{1-l_{z}^{2}}\left(\frac{d l_{z}}{d r}\right)^{2} \\
& =\left((\omega+h)^{2}-1+\frac{k_{2}}{k_{1}}\right) l_{z}-\left((\omega+h)^{2}-1+\frac{2 k_{2}}{k_{1}}\right) l_{z}^{3}+\frac{k_{2}}{k_{1}} l_{z}^{5} .
\end{aligned}
$$

Using (12) and (13) at $\delta=0, a=0$ and $\mathrm{k}_{1} \ll 1$, from (1) we obtain the following expression for the energy of PBS:

$$
\begin{aligned}
E_{s}=8 & \pi M_{0} \alpha_{x y} \sqrt{\frac{\alpha_{z}}{k_{1} B}} \\
\times \int_{0}^{\infty}\{[ & {\left[\frac{1+(\omega+h)^{2}}{2}-(\omega+h) h\right] q^{2} } \\
& \left.-\frac{k_{2}}{4 k_{1}} q^{4}+\frac{1}{2\left(1-q^{2}\right)}\left(\frac{d q}{d r}\right)^{2}+\frac{k^{2}}{2} q^{2}\right\} r^{2} d r
\end{aligned}
$$

( $M_{0}$ is the magnetization of each sublattice). The last term in this integral corresponds to the kinetic energy of PBS.

\section{Spontaneous Origin of PBS and Their Evolution into Domains of a New Phase}

As in [5], we divide the processes relating to PBS into two stages. At the first stage, the PBS originate spontaneously because of the thermal fluctuations, or by any different way, in the $\tau=0$ moment, and further evolution of PBS is carried out at the second stage. Equations (19) or (20) are used for description of the form of arose PBS. At this stage, we do not take into account the dependence of PBS parameters on the time and dissipation of energy.

PBS configurations, which are particular solutions of (19), for different $\omega$ values at $h=0.99$, and dependences 


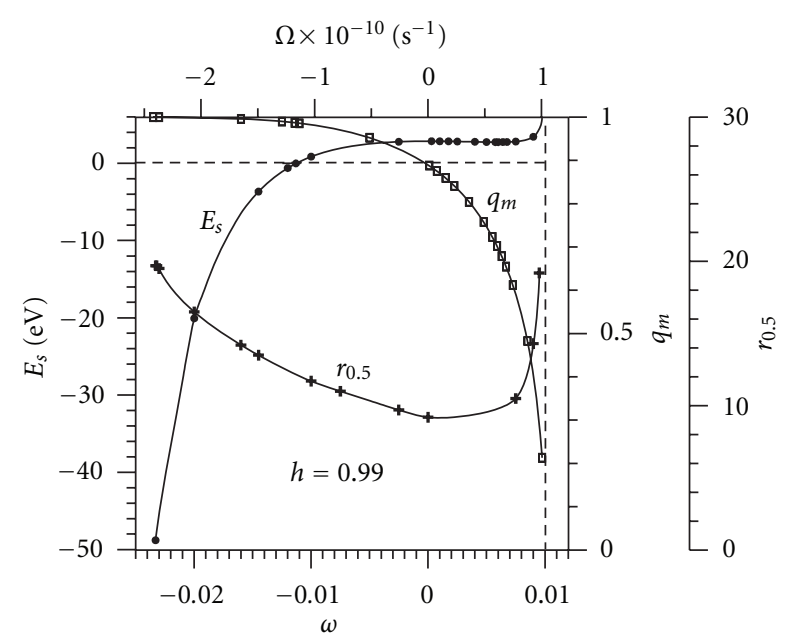

Figure 1: Frequency dependencies of the energy, amplitude, and effective radius if $h=0.99$. Here and in all subsequent figures, the values of energy are denoted by circles, the amplitude by empty squares, the frequency and the inverse of the frequency by continuous line or full squares, the radius by crosses.

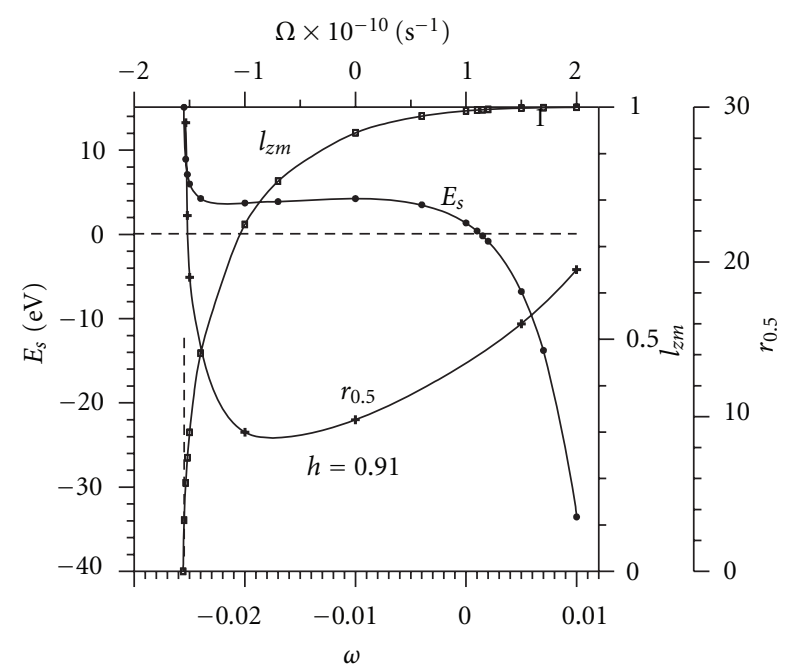

Figure 2: The same frequency dependencies as in Figure 1 for the reverse spin-flop transition, that is, at the decreasing magnetic field, if $h=0.91$.

of PBS energy and amplitudes on the precession frequency for different values of the field have been shown in [5]. In Figure 1, we present the frequency dependencies for the energy, amplitude $\left(q_{m} \equiv q(r=0)\right)$, and effective radius for PBS at $h=0.99$ only. In this case, the utmost frequency of PBS $\omega=0.01$ corresponds to the frequency of antiferromagnetic resonance, $\omega_{\text {res1 }}=(1-h)$. In Figure 2, we can see the same dependencies for the reverse spin-flop transition (at the decreasing magnetic field) at $h=0.91$. In this case, the frequency of antiferromagnetic resonance can be expressed as follows: $\omega_{\text {res } 2}=-h+\sqrt{1-k_{2} / k_{1}} \cong$ -0.01557 . Here and in all subsequent examples the following parameters typical for $\mathrm{Cr}_{2} \mathrm{O}_{3}$ have been used: $M_{0}=0.33 \times$

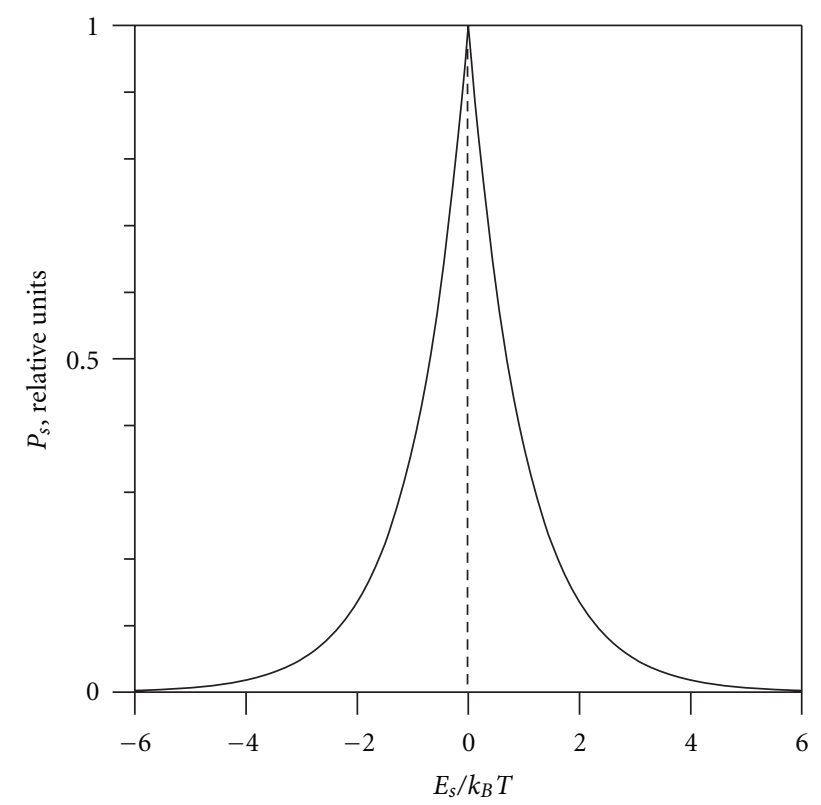

FIgURE 3: Energy dependence for probability of the spontaneous PBS creation near the point of bifurcation.

$10^{-9} \mathrm{eVOe}^{-1 \dot{A}^{-3}}, B=4.9 \times 10^{6} \mathrm{Oe}, \alpha_{x y}=\alpha_{z}=3 \times$ $10^{6} \mathrm{Oe}^{2} \dot{A}^{2}, K_{1}=700$ Oe, $K_{2}=140$ Oe, $Q=0.02$ [6].

Probability of the spontaneous creation of PBS related to the fluctuations of energy at nonzero temperatures is proportional to probability of such fluctuations. For the metastable state of our system, we use the following expression for the probability of PBS creation with the energy $E_{s}$ near the bifurcation point (see $[4,5])$ :

$$
P_{s}=A_{s} \exp \left(\frac{-\left|E_{s}\right|}{k_{B} T}\right) .
$$

Here, $A_{s}$ is the coefficient in general depending on $\omega, E_{s}$ and configuration of PBS. Generally, we have to use different configuration coefficients for positive and negative energies of PBS: $A_{s+}$ for $E_{s}>0$ and $A_{s-}$ for $E_{s}<0$. Apparently that $A_{s+}, A_{s-} \ll 1$. In Figure 3, the temperature dependence of the probability of PBS near to the bifurcation point, where the energy of PBS is near zero, is shown.

Further evolution of PBS is described by (18). As it can be seen from this equation, subsequent change of PBS configuration depends on two factors: the spatial movement of it as a whole and dissipation of energy.

In this part, we consider the change of PBS related to the dissipation only, that is, at $k \equiv 0$. Let us assume that at the moment $\tau=0$ the PBS of the $q(r, 0)$ form, corresponding to (19), have arisen. A subsequent change of PBS configuration proceeds in accordance with expression

$$
\frac{\partial q}{\partial \tau} \cong-Q k_{1} \omega(\omega+h) q\left(2 q^{2}-1\right),
$$

where $\omega=\omega(\tau)$.

In conformity with (23), the character of PBS change is determined by a sign of the precession frequency and by its amplitude. 


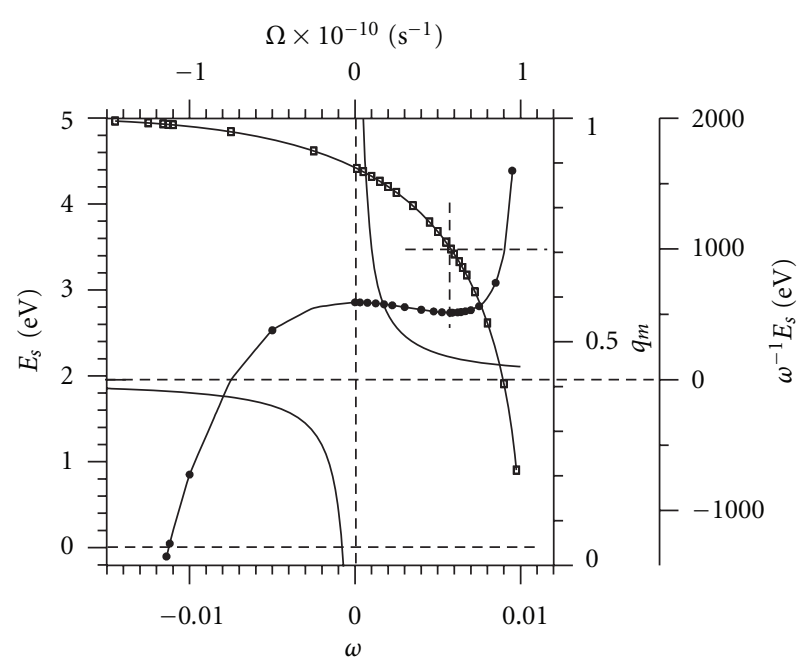

Figure 4: The frequency dependencies of energy, amplitude, and $\omega^{-1}$ value if $h=0.99$.

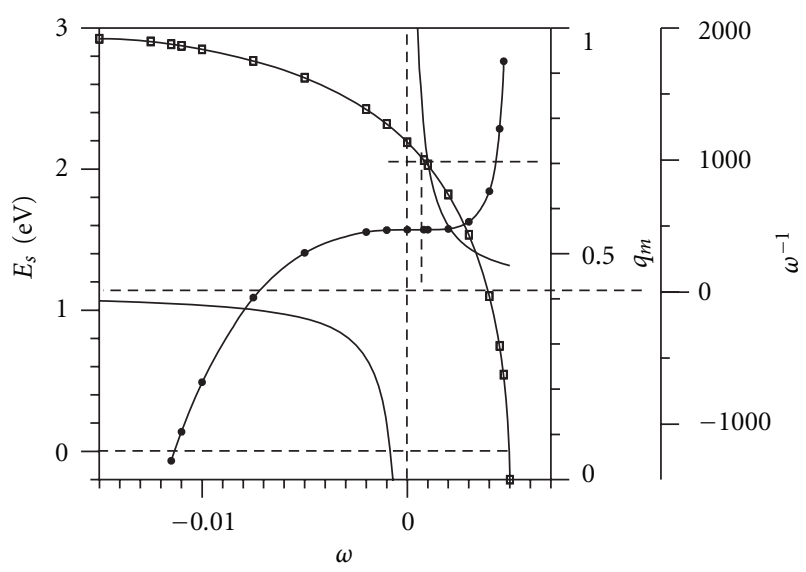

Figure 5: The same frequency dependencies as in Figure 4 if $h=$ 0.995 .

In Figures 4 to 7, several examples are adduced at $h<1$ to illustrate the PBS change because of the dissipation for different field values. If the amplitude of PBS is big enough, we can consider it as consisting of two parts: the "bulk," where value $q>\sqrt{0.5}$, and the "corona," where $q<\sqrt{0.5}$.

If $\omega_{\text {init }}<0$ and the amplitude of $\mathrm{PBS} q_{m \text {,init }}>\sqrt{0.5}$, its bulk is increasing, but the corona is decreasing. As a result, the value of $r$ corresponding to $q=\sqrt{0.5}$ is increasing, the frequency $\omega$ is increasing in absolute value, the energy is decreasing, and PBS is growing and turning into the macroscopic domain of a high-field phase. Changes of PBS can be seen at $\omega_{\text {init }}<0$ in Figure 4 and in Figure 5, at $\omega_{\text {init }}<-0.0011$ in Figure 6, and at $\omega_{\text {init }}<-0.00419$ in Figure 7. On the contrary, if $\omega_{\text {init }}>0$ and $q_{m \text {,init }}<\sqrt{0.5}$, the PBS is decreasing in amplitude. Such change can be traced in Figure 4 in the range $0<\omega<0.0058$ and in Figure 5 in the range $0<\omega<0.0008$.

If $\omega_{\text {init }}<0, q_{m \text {,init }}<\sqrt{0.5}$, the $q$ values in the bulk and corona of PBS are decreasing (see, e.g., at $0>\omega>-0.00119$

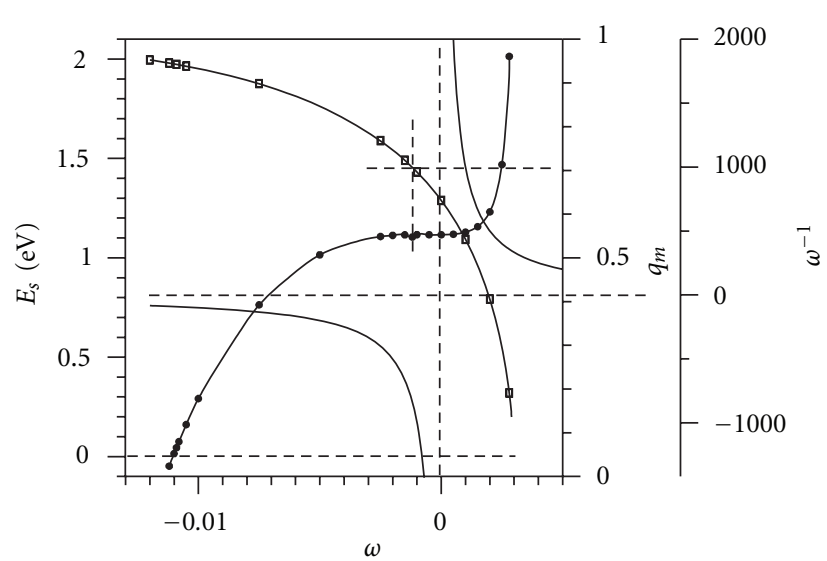

Figure 6: The same dependencies as in Figure 4 if $h=0.997$.

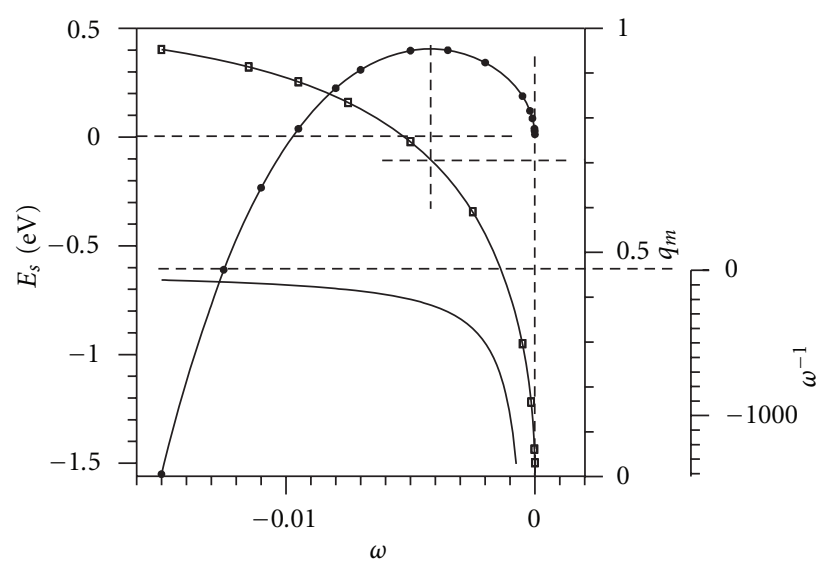

Figure 7: The same dependencies as in Figure 4 if $h=1$.

in Figure 6, and at $\omega>-0.00419$ in Figure 7). At the same time, the frequency $\omega$ is decreasing in absolute value, that is, $\omega \rightarrow-0$. In the last case, at $h=1$, the PBS disappear asymptotically, that is, $q_{m} \rightarrow 0, E_{s} \rightarrow 0$. Thus, only in cases $\omega_{\text {init }}<0, q_{m \text {,init }}>\sqrt{0.5}$ PBS are transforming into macroscopic domains of the high-field phase.

Equations (19) and (23) permit to obtain the time dependence of the frequency (and energy) due the dissipation, in accordance with the succession: $\Delta \omega \rightarrow \Delta q_{m} \rightarrow \Delta \tau \rightarrow \omega(\tau)$. For each change of the frequency $\Delta \omega$, from (19) we obtain the corresponding change of PBS amplitude $\Delta q_{m}$; further from (23), we obtain the time interval $\Delta \tau$ corresponding to this change of amplitude. As a result, we obtain the $\omega(t)$ dependences for the cases of transformation of PBS into domains of a new phase. They are presented in Figures 8 to 11 .

Figures 8 and 9 correspond to the field $h=0.99$. Initial data for PBS in Figure 8 are $\omega_{\text {init }}=-0.0025, E_{\text {init }} \cong$ $2.8553 \mathrm{eV}$, and in Figure 9: $\omega_{\text {init }}=-0.0112, E_{\text {init }} \cong$ $0.0476 \mathrm{eV}$. Note that at $T=300 \mathrm{~K}$ the average energy of the thermal fluctuations equals $k_{B} T=0.025 \mathrm{eV}$. The frequencies corresponding to value $E_{s} \cong 0$ are marked by dotted lines. Practically, the curve in Figure 9 is a part of the Figure 8 curve. 


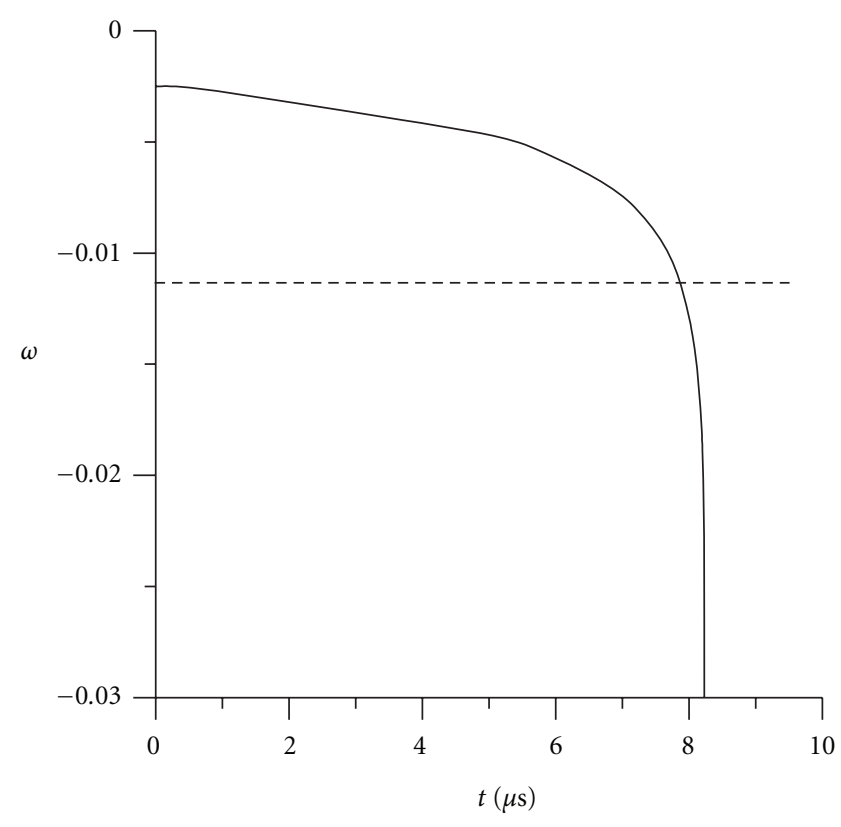

Figure 8: The time dependency of the frequency if $h=0.99$. Here the initial value of energy is $E_{\text {init }} \cong 2.855 \mathrm{eV}$ (see in Figure 4 ). The dotted line shows the frequency value corresponding to zero energy.

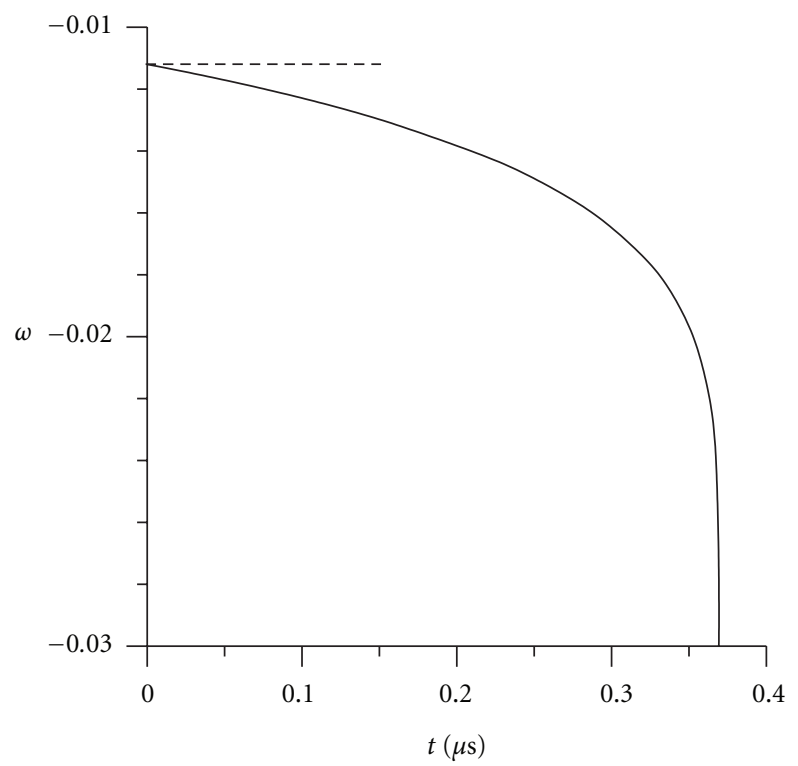

Figure 9: Time dependency of the frequency if $h=0.99$, but here $E_{\text {init }} \cong 0.0476 \mathrm{eV}$.

In Figure 10, it is shown the change of frequency for the field $h=0.997$, and in this case $E_{\text {init }}=0.2919 \mathrm{eV}$. At last, Figure 11 corresponds to $h=1, E_{\text {init }}=0.3977 \mathrm{eV}$. The four examples, in Figures 8 to 11, correspond to increase PBS and transformation of them into domains of high-field phase.

Sequence of change of the form and sizes for PBS at $h=$ 0.99, $\omega_{\text {init }}=-0.0025$ has been shown in Figure 12 (note that the similar sequences presented in [5] are wrong, since the

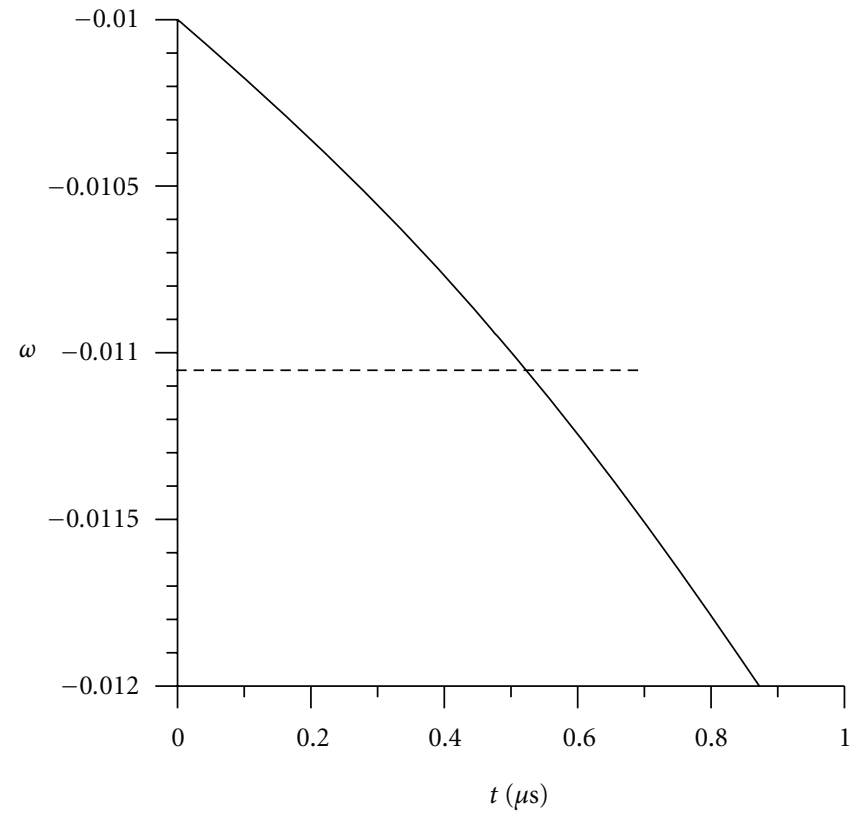

FIGURE 10: The time dependency of the frequency if $h=0.997$. Here $E_{\text {init }} \cong 0.292 \mathrm{eV}$ (see in Figure 6).

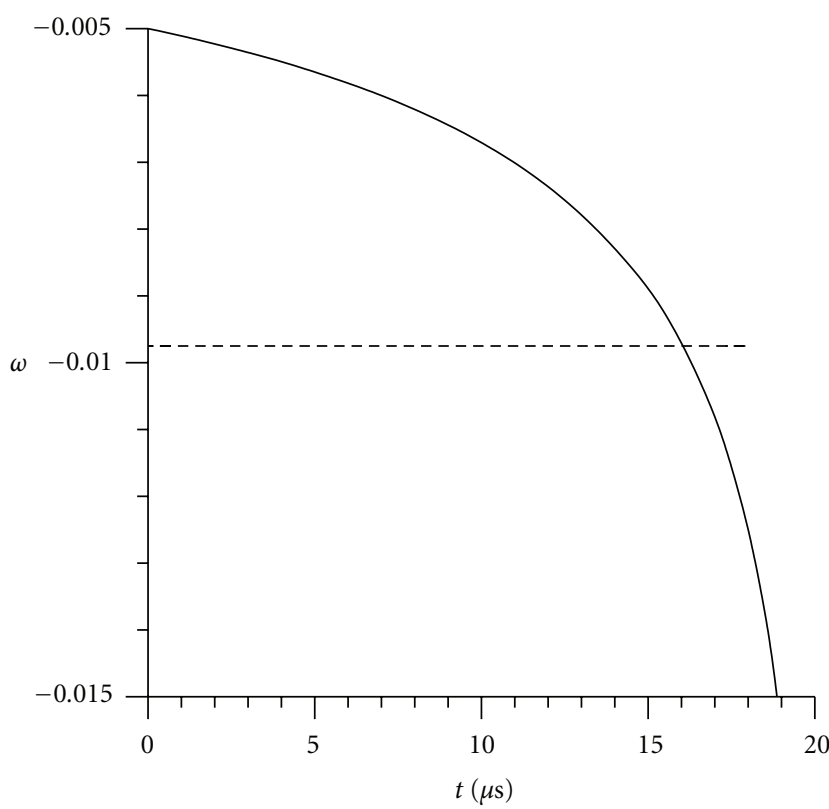

FIGURE 11: Time dependency of the frequency if $h=1$ and the initial amplitude $q_{m \text {,init }}>\sqrt{0.5}$. Here the initial parameters of PBS are the following: $E_{\text {init }} \cong 0.398 \mathrm{eV}, q_{m \text {,init }}=0.747, \omega_{\text {init }}=-0.005$ (see in Figure 7).

frequency change connected with dissipation has not been taken into consideration).

Asymptotical disappearance of PBS at $h=1$ is illustrated in Figure 13. 


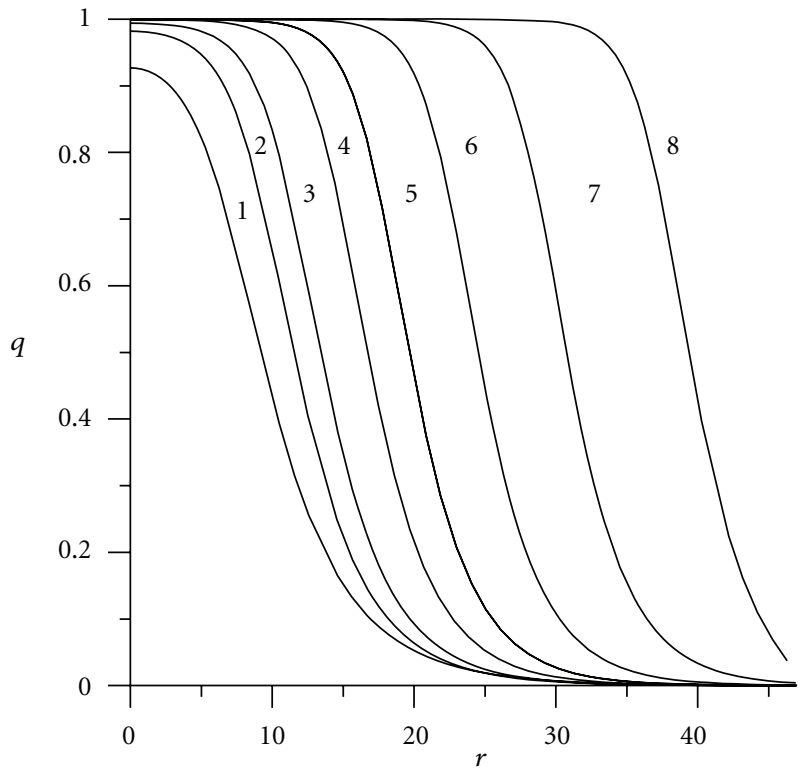

FIgURE 12: A sequence of configurations of PBS if $h=0.99$ and the initial amplitude $q_{m \text {,init }}>\sqrt{0.5}$ corresponding to Table 1 .

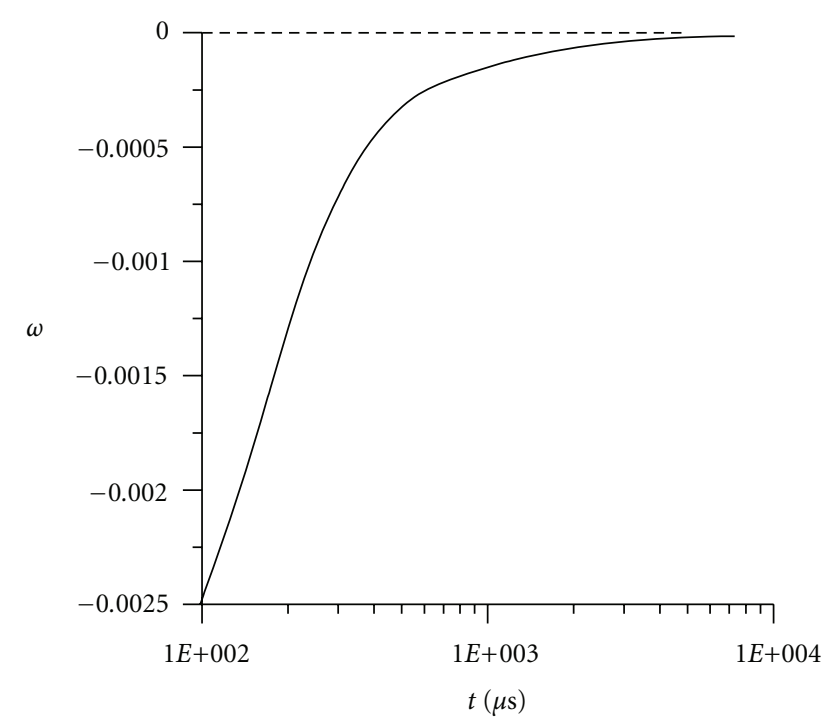

FIgURE 13: Fading of PBS if $h=1$ and the initial amplitude $q_{m \text {,init }}<\sqrt{0.5}$. Here the parameters of initial PBS are the following: $\omega_{\text {init }}=-0.0035, q_{m \text {,init }} \cong 0.666, E_{\text {init }}=0.3996 \mathrm{eV}$ (maximum energy of PBS for $h=1$ equals to $E_{s \max }=0.405465 \mathrm{eV}$, see in Figure 7).

\section{The Change of PBS during Their Movement}

Now, we consider the influence of movement on soliton form. Using (7), (9), and (10) for the $Q \omega=0$ case, we obtain the following expression:

$$
\frac{\partial q}{\partial \tau}=\sqrt{k_{1}} 2 k\left(m_{z} \frac{\partial q}{\partial x}+l_{z} \frac{\partial p}{\partial x}\right) \cong 2 k_{1} k(\omega+h)\left(3 q^{2}-1\right) \frac{\partial q}{\partial x} .
$$

TABle 1

\begin{tabular}{lcccc}
\hline No. & $t(\mu \mathrm{s})$ & $\omega$ & $E_{s}(\mathrm{eV})$ & $R_{0.5}(\dot{\AA})$ \\
\hline 1 & 0 & -0.0025 & 2.7892 & 635 \\
2 & 7.6896 & -0.01 & 0.8513 & 765 \\
3 & 8.0899 & -0.0145 & -3.6714 & 885 \\
4 & 8.2097 & -0.02 & -20.08 & 1080 \\
5 & 8.2236 & -0.0233 & -48.8 & 1290 \\
6 & 8.2243 & -0.027 & -130.7 & 1610 \\
7 & 8.2263 & -0.03 & -321.23 & 2000 \\
8 & & -0.032 & -791.8 & 2580 \\
\hline
\end{tabular}

Let us present the $q(x, y, z, \tau)$ function as $q(x, y, z, \tau)=$ $q_{s}\left(x_{s}, y, z, \tau\right)$, where $x_{s}=\left(x-v_{0} \tau\right)$. In such a case, we have

$$
-v_{0} \frac{\partial q_{s}}{\partial x_{s}}+\frac{\partial q_{s}}{\partial \tau}=-2 k_{1} k(\omega+h) \frac{\partial q_{s}}{\partial x_{s}}+6 k_{1} k(\omega+h) q^{2} \frac{\partial q_{s}}{\partial x_{s}} .
$$

If we designate the value

$$
v_{0}=2 k_{1}(\omega+h) k
$$

as the velocity of movement of a soliton as a whole, then the expression $\partial q_{s} / \partial \tau=6 k_{1} k(\omega+h) q^{2} \partial q_{s} / \partial x_{s}$ or, in spherical coordinates:

$$
\frac{\partial q_{s}}{\partial \tau}=3 v_{0} q^{2} \frac{\partial q_{s}}{\partial r_{s}} \sin \theta \cos \varphi
$$

(here $r_{s}$ is the radial coordinate in the system of moving soliton) describes the deformation of a soliton because of its movement along the $x$-axis. For considering solitons, the derivative $\partial q_{s} / \partial r<0$. Consequently, the PBS frontal side is decreasing, that is, it becomes steeper, but the back side is increasing, that is, it becomes more sloped in the same extent. Thereby, "the centre of gravity" of soliton is displaced in a direction opposite to a direction of the main movement. Thus, the form of moving soliton differs from spherical, but if $Q=0$ its energy does not change. However, the dissipation $(Q \neq 0)$ results not only in change of frequency, but also in decrease of the velocity and, accordingly, of kinetic energy:

$$
E_{\text {kinet }}=\frac{\pi M_{0} B}{k_{1}(\omega+h)^{2}}\left(\frac{\alpha}{K_{1}}\right)^{1.5} v_{0}^{2} \int q^{2} r^{2} d r .
$$

In turn, as a result of the velocity reduction, PBS asymptotically becomes more spherical in the form (more precisely, owing to anisotropy, ellipsoid of rotation).

\section{Influence of the Demagnetizing Fields and a More Correct Equation for PBS}

To estimate approximately the influence of demagnetizing field in the case of PBS, let's use the formula that concerns homogeneity magnetize sphere only:

$$
H_{\text {demag }}=-\frac{4 \pi}{3} M_{z}
$$


Corresponding to (12) and (13), magnetization of PBS equals

$$
M_{s}=2 M_{0} \sqrt{k_{1}}(\omega+h) q^{2}
$$

If to us the expression (30) for the magnetization in (29), we receive the following:

$$
h_{\mathrm{demag}} \cong-\frac{8 \pi M_{0}}{3 B}(\omega+h) q^{2}
$$

We can use this expression to estimate the value of demagnetizing field. With constants that were used in our examples, we receive $h_{\text {demag }}=-0.9 \times 10^{-3} q^{2}$, that is, the relative change of magnetic field $\Delta<10^{-3}$ (in our cases $h \cong 1$ ). Such correction does not change qualitatively the characteristics of PBS and changes them quantitatively very little. Therefore, we can neglect demagnetizing fields.

It is possible to write down a more correct equation for PBS, in comparison to (19), taking into account the additions proportional to $k_{1}$ :

$$
\begin{gathered}
\left(1+k_{1}(\omega+h)^{2} q^{2}\right)\left(\frac{d^{2} q}{d r^{2}}+\frac{2}{r} \frac{d q}{d r}\right)+\frac{q}{1-q^{2}} \\
\times\left[1+2 k_{1}(\omega+h)^{2}-k_{1}(\omega+h)^{2} q^{2}\right]\left(\frac{d q}{d r}\right)^{2} \\
=q\left(1-q^{2}\right)\left[\left(1-\frac{k_{2}}{k_{1}} q^{2}\right)\left(1+k_{1}(\omega+h)^{2} 1\left(1-q^{2}\right)\right)\right. \\
\left.\times\left(1-k_{1}(\omega+h)^{2} q^{2}\right)-(\omega+h)^{2}\right] .
\end{gathered}
$$

However, using the value $k_{1}=1.43 \times 10^{-4}$ corresponding to $\mathrm{Cr}_{2} \mathrm{O}_{3}$ [6], the solutions of (32) differ by less than $1 \%$ from the solutions of (19).

\section{Conclusions}

(1) It is shown that dissipation of energy for precessing ball solitons is accompanied by the change of the precession frequency and by the deceleration of spatial movement.

(2) Kinetics of the PBS is defined by the sign of initial precession frequency and the amplitude of the originated PBS. PBS transforms into the domains of the new phase if the initial frequency of precession is negative $\left(\omega_{\text {init }}<0\right)$ and the initial amplitude $q_{m \text {,init }}>\sqrt{0.5}$. In the paper, the whole process of such transformation of PBS has been analyzed.

(3) If $\omega_{\text {init }}<0, q_{m \text {,init }}<\sqrt{0.5}$, the $q$ values in the PBS are decreasing and frequency $\omega$ is decreasing in absolute value, $\omega \rightarrow-0$.

(4) At spatial movement of PBS, its form is deformed, but it does not change the size and the amplitude. The dissipation results not only in change of precession frequency, but also in reduction of the velocity of movement. As a result of the velocity reduction, the shape of PBS is approaching to spherical.

\section{Acknowledgment}

The author is grateful to Professor A. Zvezdin for very useful discussions about the solitonic problem.

\section{References}

[1] V. V. Nietz, "Non-linear periodic waves and solitons in an antiferromagnet with uni-axial anisotropy during a spin-flop transition," Journal of the Moscow Physical Society, vol. 9, no. 1, p. 63, 1999.

[2] V. V. Nietz, "Neutron scattering by ball solitons," in Euro-Asian Symposium "Trends in Magnetism", vol. 92 of Physics of Metals and Metallography, p. S243, Ekaterinburg, Russia, FebruaryMarch 2001.

[3] V. V. Nietz, "Neutron scattering by magnetic ball solitons," Journal of Magnetism and Magnetic Materials, vol. 266, no. 3, pp. 258-267, 2003.

[4] V. V. Nietz and A. A. Osipov, "Ball solitons in the kinetics of magnetic first-order phase transitions: a new mechanism of phase reconstruction," Crystallography Reports, vol. 53, no. 2, pp. 266-270, 2008.

[5] V. V. Nietz and A. A. Osipov, "Ball solitons in kinetics of the first-order magnetic phase transition," Journal of Magnetism and Magnetic Materials, vol. 320, no. 8, pp. 1464-1471, 2008.

[6] S. Foner, "High-field antiferromagnetic resonance in $\mathrm{Cr}_{2} \mathrm{O}_{3}$," Physical Review, vol. 130, no. 1, pp. 183-197, 1963. 

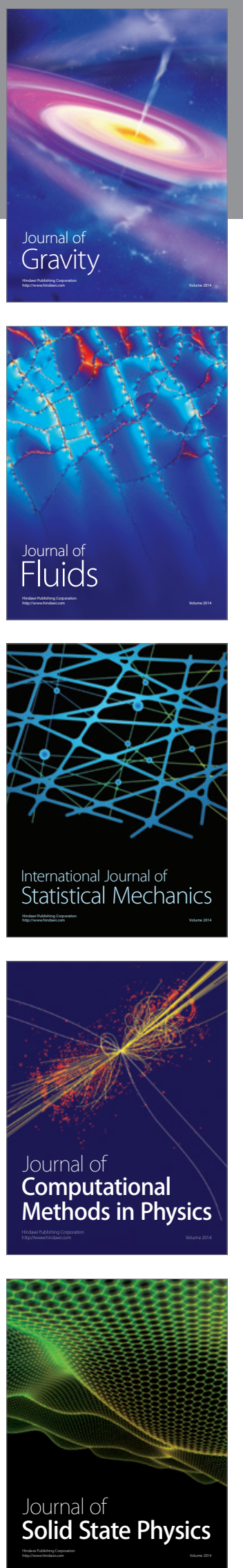

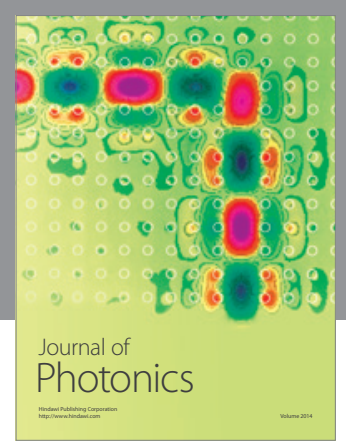

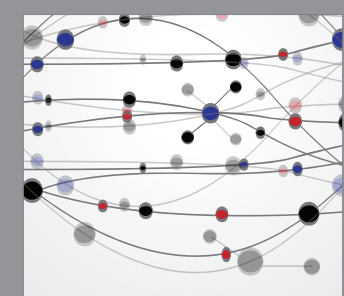

The Scientific World Journal
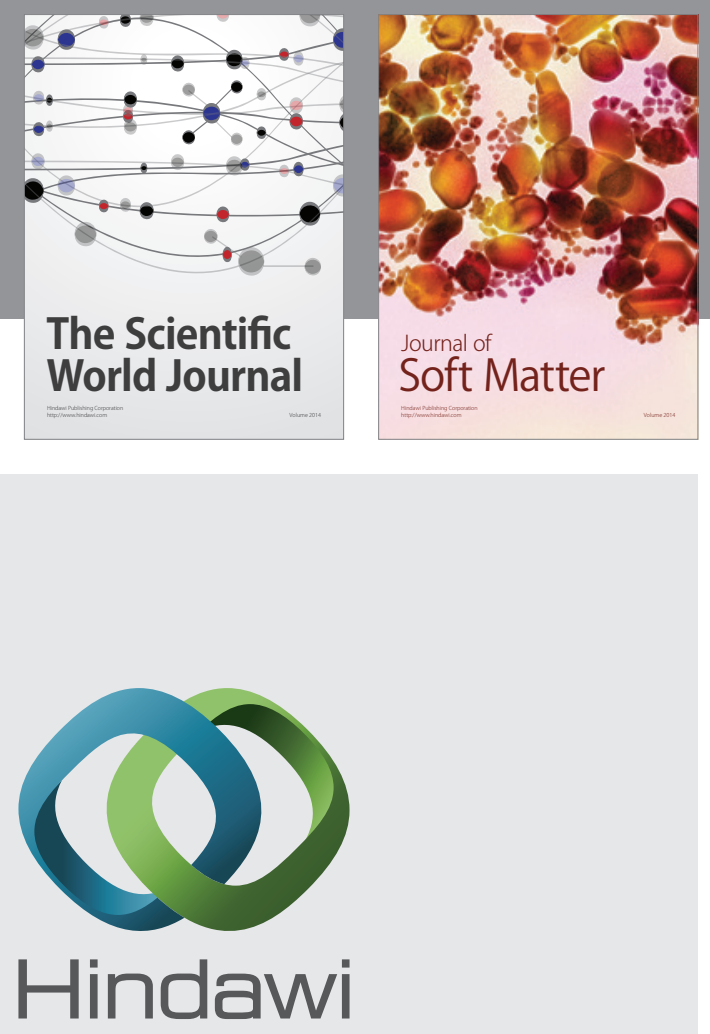

Submit your manuscripts at

http://www.hindawi.com
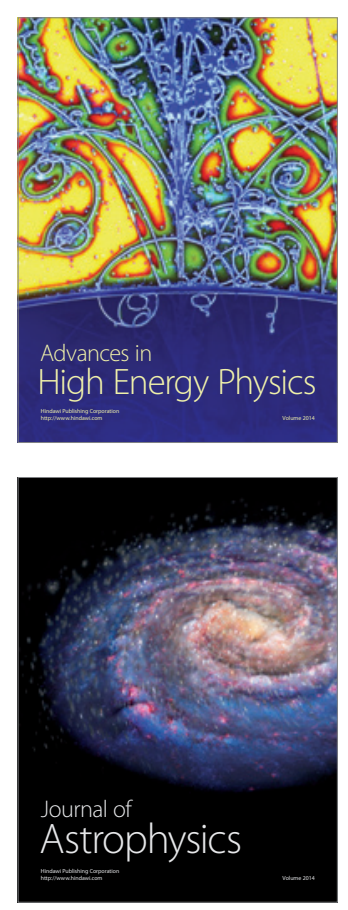
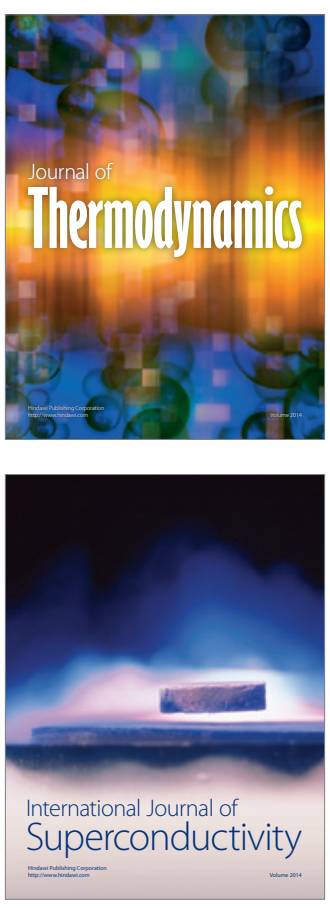
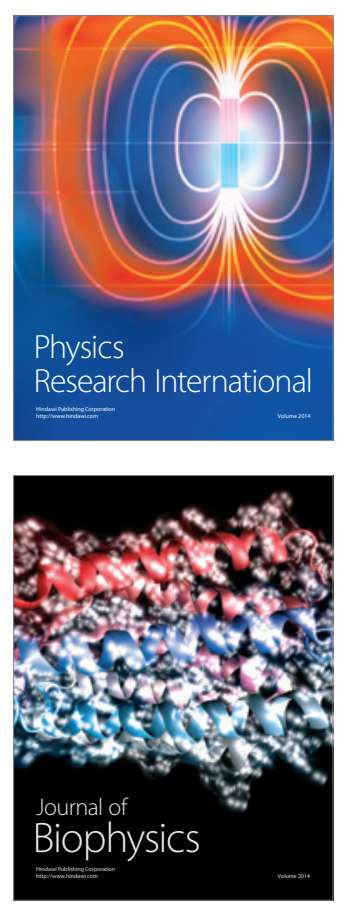
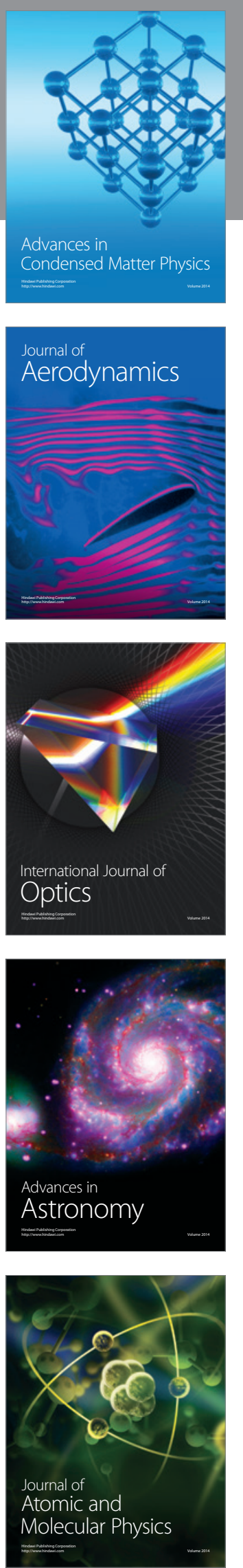